\title{
Predictions of melting, crystallization, and local atomic arrangements of aluminum clusters using a reactive force field
}

\author{
J. G. O. Ojwang,, ${ }^{1, a)}$ Rutger van Santen, ${ }^{1}$ Gert Jan Kramer, ${ }^{1}$ Adri C. T. van Duin, ${ }^{2}$ and \\ William A. Goddard III $^{3}$ \\ ${ }^{1}$ Schuit Institute of Catalysis, Eindhoven University of Technology, Postbus 513, Den Dolech 2, Eindhoven \\ $5600 \mathrm{MB}$, The Netherlands \\ ${ }^{2}$ Department of Mechanical and Nuclear Engineering, Pennsylvania State University, University Park, \\ Pennsylvania 16802, USA \\ ${ }^{3}$ Materials and Process Simulation Center (MSC), California Institute of Technology, 1200 East California \\ Boulevard, Pasadena, California 91125, USA
}

(Received 5 September 2008; accepted 25 November 2008; published online 30 December 2008)

\begin{abstract}
A parametrized reactive force field model for aluminum $\operatorname{ReaxFF}_{\mathrm{Al}}$ has been developed based on density functional theory (DFT) data. A comparison has been made between DFT and ReaxFF $\mathrm{Al}$ outputs to ascertain whether ReaxFF $\mathrm{Al}_{\mathrm{Al}}$ is properly parametrized and to check if the output of the latter has correlation with DFT results. Further checks include comparing the equations of state of condensed phases of $\mathrm{Al}$ as calculated from DFT and $\mathrm{ReaxFF}_{\mathrm{Al}}$. There is a good match between the two results, again showing that ReaxFF ${ }_{\mathrm{Al}}$ is correctly parametrized as per the DFT input. Simulated annealing has been performed on aluminum clusters $\mathrm{Al}_{n}$ using $\mathrm{ReaxFF}_{\mathrm{Al}}$ to find the stable isomers of the clusters. A plot of stability function versus cluster size shows the existence of highly stable clusters (magic clusters). Quantum mechanically these magic clusters arise due to the complete filling of the orbital shells. However, since force fields do not care about electrons but work on the assumption of validity of Born-Oppenheimer approximation, the magic clusters are therefore correlated with high structural symmetry. There is a rapid decline in surface energy contribution due to the triangulated nature of the surface atoms leading to higher coordination number. The bulk binding energy is computed to be $76.8 \mathrm{kcal} / \mathrm{mol}$. This gives confidence in the suitability of ReaxFF for studying and understanding the underlying dynamics in aluminum clusters. In the quantification of the growth of cluster it is seen that as the size of the clusters increase there is preference for the coexistence of fcc/hcp orders at the expense of simple icosahedral ordering, although there is some contribution from distorted icosahedral ordering. It is found that even for aluminum clusters with 512 atoms distorted icosahedral ordering exists. For clusters with $N \geq 256$ atoms fcc ordering dominates, which implies that at this point we are already on the threshold of bulklike bonding. (C) 2008 American Institute of Physics. [DOI: 10.1063/1.3050278]
\end{abstract}

\section{INTRODUCTION}

Small aluminum clusters have been studied extensively with a view to getting a better understanding of atomic aggregates of aluminum, including the threshold of cluster-bulk interface. ${ }^{1,2}$ Previous works on aluminum clusters have addressed issues such as magnetic properties, ${ }^{3}$ static polarizabilities of $\mathrm{Al}_{n},{ }^{4}$ ionization thresholds, and reactivities. Other theoretical computations ${ }^{6-11}$ have tackled issues to do with the energetically lowest structures of small aluminum clusters, although even for small clusters such as $\mathrm{Al}_{4}, \mathrm{Al}_{5}$, and $\mathrm{Al}_{7}$ there are still lingering uncertainties on the preferred configurations. The other point of interest is the existence of magic clusters (superatoms) of aluminum. Superatoms are clusters of atoms that exhibit some of the properties of elemental atoms. For instance, $\mathrm{Al}_{7}$ and $\mathrm{Al}_{13}$ have been found to behave like superatoms. $\mathrm{Al}_{7}$ behaves like an alkali since it has 21 valence electrons, while $\mathrm{Al}_{13}$, which has 39 valence electrons, behaves like a halogen. Studies have been con-

${ }^{a)}$ Electronic mail: j.g.o.ojwang@tue.nl. ducted on the reactions of aluminum clusters with oxygen and it was observed that $\mathrm{Al}_{7}^{+}, \mathrm{Al}_{13}^{-}$, and $\mathrm{Al}_{23}^{-}$do not react with oxygen. ${ }^{12}$ This suggested that these clusters were stable entities due to their closed electronic shells with 20,40, and 70 electrons for $\mathrm{Al}_{7}^{+}, \mathrm{Al}_{13}^{-}$, and $\mathrm{Al}_{23}^{-}$, respectively. These magic clusters should perhaps serve as the zone centers for crystal formation. Atoms agglomerate to form superatoms, which then coalesce to form crystals. Using a glue potential, ${ }^{13}$ Doye ${ }^{14}$ investigated the stabilities of aluminum clusters up to $\mathrm{Al}_{190}$ and found a series of magic clusters starting from $\mathrm{Al}_{13}$, $\mathrm{Al}_{19}, \mathrm{Al}_{23}, \ldots, \mathrm{Al}_{55}, \ldots$. This elegant approach was purely based on geometrical structures of the aluminum clusters as the higher the symmetry the more stable the structure.

Even more intriguing is the melting of smaller clusters. It is not always the case that small clusters have lower melting point than the bulk. It was found in the case of gallium and tin that the melting point of small clusters is higher than that of the bulk due to differing structures and stronger bonding in comparison to the bulk structures. ${ }^{15-18}$ Such intrigues make understanding of the dynamics governing cluster formation, magic numbers, and melting an important key into 
unraveling how matter behaves in such small regimes. Clusters are the building blocks of bulk systems. Aluminum atoms must first agglomerate to form clusters. These clusters can then fuse together to form crystals. Studies of aluminum clusters will therefore shed some more light on the macroscopic evolution of the molecular phase to condensed matter realms with the increase in the number of aluminum atoms.

In studying aluminum clusters a choice must be made between accuracy and computational efficiency. Traditionally density functional theory (DFT) is the tool of choice for computational physicists/chemists especially in the condensed matter realm. ${ }^{19}$ However, this is against a backdrop of enormous computational demands. For instance, optimization of $\mathrm{Al}_{19}$ cluster in a cubic cell of side $20 \AA^{3}$ at the $\Gamma$ point using a plane waves cutoff of $600 \mathrm{eV}(1 \mathrm{eV}$ $=23.06 \mathrm{kcal} / \mathrm{mol}$ ) in VASP (Ref. 20) on amd64 processors (with eight nodes) took about $67 \mathrm{~h}$. In contrast, using a reactive force field (ReaxFF) this optimization was done in a fraction of a second. One must therefore take cognizance of the fact that a more robust approach is to use a force field without necessarily sacrificing accuracy at the altar of computational efficiency. Our goal in this project was twofold. First, we wanted to show that ReaxFF, which has been successfully used to accurately predict the dynamical and reactive processes in hydrocarbons, ${ }^{21}$ silicon/silicon oxides, ${ }^{22}$ aluminum/aluminum oxides, ${ }^{23}$ nitramines,${ }^{24}$ and sodium hydride, ${ }^{25}$ can also be used to predict the properties of metallic systems. Second, we wanted to have a better understanding on the nature of phase transition in the ordering of atoms as the size of the cluster increases. To achieve these goals we parametrized the reactive force field $\left(\operatorname{ReaxFF}_{\mathrm{Al}}\right)$ so that it could aptly capture the nature of chemical bonding in aluminum and then use the parametrized force field to do molecular dynamics (MD) simulations on clusters of aluminum. $\operatorname{ReaxFF}_{\mathrm{Al}}$ was then used to study the energetics and conformations of small aluminum clusters, simulate melting and crystallization of larger clusters, and study the local atomic ordering of clusters during crystallization. In particular, we examine the transition from icosahedral ordering to fcc ordering. We have used potential energy and heat capacity to characterize melting in the aluminum clusters. A Honeycutt-Andersen (HA) pair analysis ${ }^{26}$ was used to discriminate between icosahedral and fcc ordering of aluminum clusters, starting with $\mathrm{Al}_{256}$ cluster and ending with $\mathrm{Al}_{3072}$ cluster.

This paper is organized as follows. Section II deals with force field parametrization and simulation methods while Sec. III, which is the discussion section, focuses on the results for melting, crystallization, and local atomic arrangements of aluminum clusters. The conclusion is detailed in Sec. IV.

\section{COMPUTATIONAL METHODS}

\section{A. Force field parametrization and validation}

$\operatorname{ReaxFF}_{\mathrm{Al}}$ has been parametrized in line with the methodology used to develop ReaxFF $\mathrm{NaH}_{\mathrm{N}}$ (Ref. 25) and $\mathrm{ReaxFF}_{\mathrm{MgH}}{ }^{27}$ ReaxFF does not use fixed connectivity assignment between atoms but rather deploys the bond-order formalism, which allows for bond breaking and formation in line with the works of Tersoff ${ }^{28}$ and Brenner. ${ }^{29}$ The bond order is directly determined from the instantaneous interatomic distance $r_{i j}$, which is updated per every iteration. Implemented in ReaxFF is the electronegativity equalization method $^{30}$ used to calculate the distribution of charges. Since charges are updated per every iteration, it implies that the nonuniform distribution of charges in small clusters, which emanates from large variations in coordination of atoms and therefore large differences in charges in various parts of the cluster, is correctly treated.

The fitting data used in ReaxFF were obtained from high level quantum mechanical calculations using VASP, which implements a projector augmented plane wave method approach. ${ }^{31}$ For all calculations a plane wave cutoff of 600 $\mathrm{eV}$ was used. The Kohn-Sham ground state is selfconsistently determined in an iteration matrix diagonalization scheme. The calculations used the generalized gradient approximation of Perdew and Wang ${ }^{32-34}$ (GGA-PW91) to represent electronic-correlation effects for a particular ionic configuration. For cluster calculation a cubic supercell of side $20 \AA$ (which ensured that interaction between clusters in adjacent cells is negligible) was used with the cluster/ molecule placed at the center of the cell. The Brillouin zone was then sampled at the $\Gamma$ point.

For the condensed phases, for all volumes of the structures considered, the structures were fully optimized using force as well as stress minimization. The ions involved are steadily relaxed toward equilibrium until the HellmanFeynman forces are minimized to less than $10^{-4} \mathrm{eV} / \AA$ with conjugate gradient algorithm during all relaxation runs. A convergence of $10^{-6} \mathrm{eV} /$ atom was placed as a criterion on the self-consistent convergence of the total energy. Brillouin zone integrations were performed using the following $k$ points: Al-fcc $(15 \times 15 \times 15)$, Al-bcc $(19 \times 19 \times 19)$, Al-hcp $(15 \times 15 \times 15)$, Al-simple cubic $(\mathrm{sc})(15 \times 15 \times 15)$, and Aldiamond $(10 \times 10 \times 10)$ as per the Monkhorst-Pack grid procedure. ${ }^{35}$ The reference configurations for valence electrons used were $\mathrm{Al}\left(3 s^{2} 3 p^{1}\right)$. In determining the equilibrium volume, for a fixed cell volume of each structure, the cell shape and atomic coordinates were fully optimized until the forces were less that $10^{-4} \mathrm{eV} / \AA$ atom. The structure with the lowest energy was determined by plotting a total energy versus cell-volume curves for all the structures considered. The obtained energies were fitted to a Birch-Murnaghan equation of state (EoS) (Ref. 36) in order to get the equilibrium volume and minimum energy. The final structure was then determined by optimizing the lattice parameters and atomic positions at this equilibrium volume until the forces on the ions were less than $10^{-4} \mathrm{eV} / \AA$ atom.

ReaxFF energy expressions were parametrized by fitting to a training set containing the DFT derived EoS of pure $\mathrm{Al}$ phases, reaction energies, and bond dissociation profiles on small finite clusters. The bond and atom parameters for the ReaxFF energy functions (Tables I and II) were determined from the equations of state and cohesive energies of Al-metal condensed phases. The symbols and meanings of the parameters in Tables I-III are shown in Refs. 22 and 24. 
TABLE I. Bond energy and bond-order parameters. $D_{\mathrm{e}}^{\sigma}$ is in $\mathrm{kcal} / \mathrm{mol}$.

\begin{tabular}{lccccc}
\hline \hline Bond & $D_{\mathrm{e}}^{\sigma}$ & $P_{\mathrm{be}, 1}$ & $P_{\mathrm{be}, 2}$ & $P_{\mathrm{bo}, 1}$ & $P_{\mathrm{bo}, 2}$ \\
\hline $\mathrm{Al}-\mathrm{Al}$ & 34.1 & 0.4832 & 6.4631 & -0.15 & 6.160 \\
\hline \hline
\end{tabular}

\section{B. Simulation, thermodynamic, and structural analysis methods}

The MD calculations were done using a velocity Verlet algorithm ${ }^{37}$ to integrate Newton's equations of motion. The simulations were performed in the canonical ensemble, $N V T$ (constant number of particles, volume, and temperature). The time step used for all simulations was 1.0 fs. This led to stable dynamics trajectories. The original clusters were built up from geometries constructed from fcc blocks. The clusters were then minimized to remove bad contact angles and then equilibrated at $300 \mathrm{~K}$, followed by annealing to $0 \mathrm{~K}$. The annealed structures were then heated up to desired temperatures in order to determine their global minima. Determining the global minima for clusters using simulated annealing is a delicate task since there are bound to be several "deep" local minima in the potential energy hypersurfaces. Merely equilibrating the structure at a particular temperature can lead to the system being trapped in a local minimum. To circumvent this problem we used a slow heating rate, which enabled the system to have enough time to sample the various possible conformations in the phase space and wring itself out of the undesirable deep local minima. Careful analysis and tests showed that a heating rate of $2.5 \times 10^{9} \mathrm{~K} / \mathrm{s}$ was capable of predicting the most stable isomers of small aluminum clusters while if a heating rate of $2.5 \times 10^{10} \mathrm{~K} / \mathrm{s}$ was used some of these isomers were missed during the simulated annealing process. For instance, at a heating rate of $2.5 \times 10^{10} \mathrm{~K} / \mathrm{s}$ we could not capture the stable isomer of $\mathrm{Al}_{10}$ but when the heating rate was reduced to $2.5 \times 10^{9} \mathrm{~K} / \mathrm{s}$ it was captured by the force field. Therefore it is important to use a well optimized heating rate so as to avoid being trapped in an energetically unfavorable deep local minimum. For large clusters $(N>200$ atoms $)$ we had to use even a lower heating rate, $2.5 \times 10^{8} \mathrm{~K} / \mathrm{s}$, to capture the global minima. We shall return to this point in Sec. III.

The heat capacity was calculated by fitting smooth cubic splines to the average potential energy during the heating process. The heat capacity is the temperature derivative of the potential energy as follows:

$$
C_{p}(T)=\frac{d(P E)}{d T}+\frac{3 R}{2},
$$

where $R=1.9872159 \mathrm{cal} \mathrm{K}^{-1} \mathrm{~mol}^{-1}$ is the molar gas constant. With this in mind the melting point is defined as the temperature with the maximum apparent heat capacity, which is caused by a sharp increase in the mobility of atoms

TABLE II. Atom parameters.

\begin{tabular}{lcc}
\hline \hline Atom & $p_{\text {ov } / \text { un }}$ & $\lambda_{11}$ \\
\hline $\mathrm{Al}$ & -23.18 & 4.50 \\
\hline \hline
\end{tabular}

TABLE III. van der Waals parameters and bond radius parameters.

\begin{tabular}{lcccc}
\hline \hline Atoms & $\begin{array}{c}r^{\sigma} \\
(\AA)\end{array}$ & $\begin{array}{c}r_{\mathrm{vdW}} \\
(\AA)\end{array}$ & $\begin{array}{c}E_{\mathrm{vdW}} \\
(\mathrm{kcal} / \mathrm{mol})\end{array}$ & $\begin{array}{c}\gamma_{\mathrm{vdW}} \\
(\AA)\end{array}$ \\
\hline $\mathrm{Al}-\mathrm{Al}$ & 2.1322 & 2.2966 & 5.364 & 3.104 \\
\hline \hline
\end{tabular}

in the system. However, for clusters it becomes problematic to locate the exact melting point due to the premelting of the surface of the cluster. This leads to a temperature region in which both the liquid and solid phases coexist (dynamic coexistence melting). Since phase transformation in a material is accompanied by an increase in vibrational motion, an alternative way of distinguishing the solid-liquid-like phase transition is to use the Lindemann index, which measures the vibrational motion of atoms. The Lindemann concept ${ }^{38}$ has it that melting occurs when the stretching curve gets unstable second derivatives. It can be taken to be a measure of dynamic disorder in a material. The Lindemann index is at times equated to the relative root-mean-square $(\mathrm{rms})$ bond length fluctuations, which are expressed as

$$
\delta=\frac{2}{N(N-1)} \sum_{i, j(i \neq j)}^{N} \frac{\sqrt{\left\langle r_{i j}^{2}\right\rangle_{T}-\left\langle r_{i j}\right\rangle_{T}^{2}}}{\left\langle r_{i j}\right\rangle_{T}},
$$

where $\langle. .\rangle_{T}$ denotes the thermal average at temperature $T$ and $r_{i j}$ is the distance between atoms $i$ and $j$. During melting the Lindemann index is expected to increase abruptly by a factor of more than 3. Usually in bulk materials $\delta<0.1$ indicates a solid phase. For clusters, due to surface effects, $\delta<0.08$ is an indication of a solid phase. An extensive analysis on determination of heat capacity and rms bond length fluctuations for clusters is given in Ref. 39. Further insight into the structure of clusters has been done by analyzing the radial distribution function $g(r)$, which describes how the atoms are radially packed around each other and shows the structural ordering of the atoms in a system. $g(r)$ is linked to the experimentally observable structure factor $S(k)$ obtained in diffraction experiments.

To understand the phenomenon of icosahedral to fcc growth we studied the local atomic arrangements in four clusters of aluminum, viz., $\mathrm{Al}_{256}, \mathrm{Al}_{512}, \mathrm{Al}_{1024}$, and $\mathrm{Al}_{3072}$. The structures were first heated up to temperatures between 250 and $2000 \mathrm{~K}$ and then annealed to $0 \mathrm{~K}$ at a rate of 2.5 $\times 10^{9} \mathrm{~K} / \mathrm{s}$. HA pair analysis was then performed on the clusters during the annealing process in order to ascertain at which cluster size fcc ordering became more important with respect to icosahedral ordering. In HA pair analysis the local structure surrounding a pair of atoms (root pair) is classified using four indices $(\mathrm{klmn})$ as follows.

(1) The first index $k$ is 1 when the atoms in the root pair are bonded, else it is 2 .

(2) The second index $l$ is the number of near-neighbor atoms common to the root pairs.

(3) The third index $m$ is the number of nearest neighbor bonds between the shared neighbors.

(4) The fourth index $n$ is used to differentiate between cases in which the first three indices are the same but bond geometries differ like in the cases of fcc and hcp. 


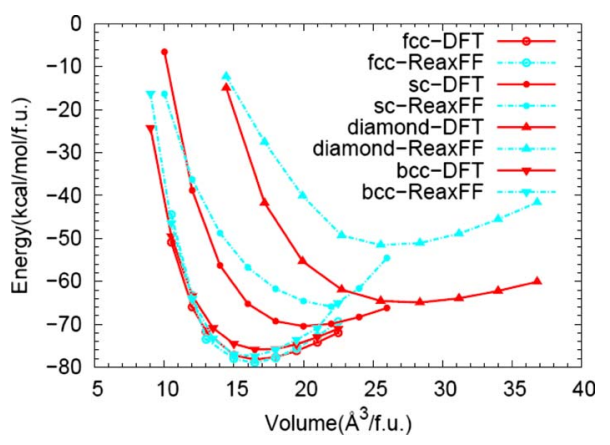

FIG. 1. (Color online) Relative stability of the various phases of aluminum as computed by DFT and ReaxFF.

The following holds in HA pair analysis prescription.

(1) The 1421 pairs represent fcc ordering, while the hcp ordering is represented by both 1421 and 1422 pairs.

(2) The 1441 and 1661 pairs represent bcc ordering, while the $1202,1311,1301,1331$, and 1321 represent rhombohedral ordering.

(3) The 15's pairs (e.g., 1551 and 1541 pairs) 1321 and 2331 are indicative of rapidly quenched liquid or amorphous states and lead to simple icosahedral ordering. In particular, the 1551 pairs represent two neighboring atoms with five common neighbors that form a bonded pentagon. Thus the 1551 pairs, which are situated in a fivefold symmetry environment, are characteristic of icosahedral ordering. The 143's pairs lead to distorted icosahedra (Ref. 40), which can also be considered as $+72^{\circ}$ disinclinations. ${ }^{41}$

Since the interest here is to understand the underlying mechanism governing transition from icosahedral structure to fcc structure, only the $1421,1422,1431$, and 1551 pairs have been considered in this work. We take $R_{\text {cut }}=3.0 \AA$, which is just slightly larger than the DFT bulk value of aluminum-aluminum bond length $(2.864 \AA)$ and is the value of the first peak in $g(r)_{\mathrm{Al}-\mathrm{Al}}$. The HA pair fractions are highly dependent on the value of $R_{\text {cut }}$, and therefore it is not possible to make a quantitative comparison with other studies but a qualitative comparison holds.

\section{RESULTS AND DISCUSSION}

\section{A. Heats of formation and geometry of clusters}

The ability of ReaxFF to capture the relative stability of condensed phases of aluminum was tested against a number of $\mathrm{Al}$ crystal modifications. For each and every phase of $\mathrm{Al}-\mathrm{metal}$ (fcc-Al, sc-Al, bcc-Al, hcp-Al, and diamond-Al) considered in this work, the quantum energies were computed for a broad range of volume, describing both expansion and compression. In Fig. 1, we see that ReaxFF correctly describes the relative phase stabilities of four phases of aluminum metal with respect to the quantum values.

The hcp phase is not included since with ReaxFF the hcp and fcc phases get the same energy; the method does not have the multibody terms for metals that are needed to distinguish these phases. For the most stable fcc phase ReaxFF

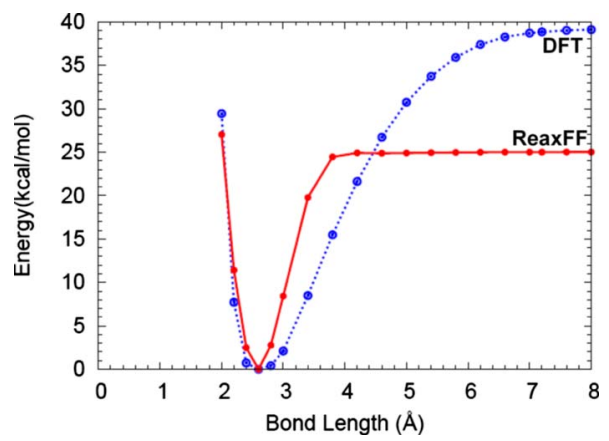

FIG. 2. (Color online) Bond dissociation profile of $\mathrm{Al}_{2}$ dimer as computed by DFT and ReaxFF. The energies were computed with reference to the equilibrium bond length's energy.

gives the equilibrium energy to be $-78.97 \mathrm{kcal} / \mathrm{mol}$ per formula unit whereas DFT gives $-78.22 \mathrm{kcal} / \mathrm{mol}$ f.u. Thus ReaxFF value differs from DFT by $0.75 \mathrm{kcal} / \mathrm{mol}$ f.u. The experimental lattice parameter is $4.0494 \AA$, while DFT gives $4.0498 \AA$ and ReaxFF gives $4.250 \AA$.

Another important comparison is on surface energy, which gives a measure of the energy needed to cleave a surface. To obtain the surface energy a five layered 20 atom slab with a vacuum layer equivalent to five layers was used. Brillouin zone was sampled using a well converged $9 \times 9$ $\times 1 k$ point. The surface energy was then calculated by comparing the total energy of bulk and slab models as follows:

$$
E^{\text {surface }}=\frac{1}{2 A}\left(E^{\text {slab }}-N E^{\text {bulk }}\right),
$$

where $E^{\text {slab }}$ is the energy of an N-layer slab, $E^{\text {bulk }}$ is the bulk energy per formula unit, and $A$ is the bulk surface area. From ReaxFF the surface energy for Al (111) surface was computed to be $1.38 \times 10^{-4} \mathrm{kcal} / \mathrm{m}^{2}$, which is in good agreement with the DFT value of $1.87 \times 10^{-4} \mathrm{kcal} / \mathrm{m}^{2}$.

The Al-Al bond energy in ReaxFF was optimized using DFT derived values of bond dissociation profile of $\mathrm{Al}_{2}$ dimer and other small aluminum clusters. Figure 2 shows the bond dissociation curve of $\mathrm{Al}_{2}$.

The dissociation curves were constructed from the equilibrium geometry using single point calculations by changing the bond length. ReaxFF gives an equilibrium bond length of $2.6 \AA$, which is the same as DFT value. These values are in good agreement with the experimental $\mathrm{Al}_{2}$ dimer bond length of $2.47 \AA .^{42}$ Only the triplet values are shown because the singlet values are energetically unfavorable throughout the dissociation curve.

In computing the free energies of the aluminum clusters, spin polarization was taken into account. It was found that in most cases the electronic configuration that favored the lowest spin multiplicity was not necessarily the most stable. For instance, for $\mathrm{Al}_{3}$ the spin doublet case was $2.07 \mathrm{kcal} / \mathrm{mol}$ more stable than the spin quartet, and for $\mathrm{Al}_{7}$ the doublet configuration was $24.8 \mathrm{kcal} / \mathrm{mol}$ more stable than the quartet arrangement. In the case of $\mathrm{Al}_{2}$, the triplet state was found to be more stable than the singlet state by $7.76 \mathrm{kcal} / \mathrm{mol}$, while for $\mathrm{Al}_{6}$ the triplet state was energetically more stable than the singlet state by $3.59 \mathrm{kcal} / \mathrm{mol}$. For $\mathrm{Al}_{4}$ the triplet state was 


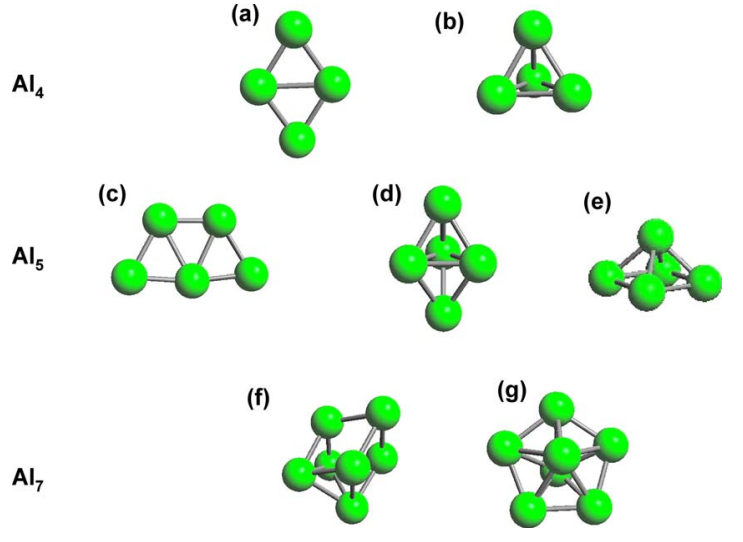

FIG. 3. (Color online) Small representative isomers of $\mathrm{Al}_{4}, \mathrm{Al}_{5}$, and $\mathrm{Al}_{7}$ clusters as predicted by DFT and ReaxFF. DFT predicts that structures a, c, and $\mathrm{f}$ are the most stable configurations for $\mathrm{Al}_{4}, \mathrm{Al}_{5}$, and $\mathrm{Al}_{7}$, respectively. ReaxFF, on the other hand, predicts that structures $b, d$, and $g$ are the most stable configurations for $\mathrm{Al}_{4}, \mathrm{Al}_{5}$, and $\mathrm{Al}_{7}$, respectively.

more stable than the singlet state by $3.5 \mathrm{kcal} / \mathrm{mol}$. For $\mathrm{Al}_{11}$, it was found that the spin doublet state was more stable than the spin quartet state by $9.17 \mathrm{kcal} / \mathrm{mol}$.

DFT calculation shows that the most stable form of $\mathrm{Al}_{3}$ is an equilateral triangle, in agreement with the work of Pettersson et al. ${ }^{1}$ For $\mathrm{Al}_{4}$ the planar rhombus $\left(D_{2 h}\right)$ conformation is found to be more stable than the pyramidal form $\left(C_{3 v}\right)$, in agreement with Koutecky et al. ${ }^{43}$ and Pettersson et al. ${ }^{1}$ In the case of $\mathrm{Al}_{5}$, Jug et al. ${ }^{44}$ found the pyramidal form to be the most stable, whereas Petterson et al. ${ }^{1}$ and Yang et $a l .{ }^{9}$ found the planar $\left(C_{2 v}\right)$ form to be more stable than the pyramidal form. The DFT calculations for $\mathrm{Al}_{5}$ are consistent with the works of Yang et al. ${ }^{9}$ In addition, Pettersson et al. ${ }^{1}$ found that in the case of $\mathrm{Al}_{6}$ the octahedron is the most stable form, whereas Upton ${ }^{2}$ found a distorted octahedron to be the most stable. Clearly, for Al clusters with more than five atoms three dimensional (3D) structures are favored since as the size of the clusters increases the number of nearest neighbors also increases, but this cannot be achieved in a planar structure. For instance, the icosahedral arrangement of $\mathrm{Al}_{13}$ enables the atom in the middle of the structure to have 12 neighbors. There are interesting differences between ReaxFF and DFT predictions, as shown in Fig. 3.

For instance, for $\mathrm{Al}_{4}$, DFT favors the rhombus structure [Fig. 3(a)] in agreement with Koutecky et al., ${ }^{43}$ Pettersson, ${ }^{1}$ Bauschlicher and Pettersson ${ }^{45}$ and Jones. ${ }^{46}$ ReaxFF, on the other hand, finds a tetrahedron structure [Fig. 3(b)] to have the lowest energy. Upon heating the rhombus structure it immediately transforms into the tetrahedron isomer even at temperatures of as low as $1 \mathrm{~K}$. The tetrahedron structure was also found by Böyükata and Güvenç, ${ }^{47}$ who used the embedded atom method (EAM) ${ }^{48}$ to be the most stable isomer of $\mathrm{Al}_{4}$. For $\mathrm{Al}_{5}$ DFT favors the planar rhombus $\left(C_{2 v}\right)$-like structure [Fig. 3(c)], in agreement with Pettersson et al. ${ }^{1}$ but ReaxFF finds the trigonal bipyramidal $\left(D_{3 h}\right)$ isomer [Fig. 3(d)] to be the more stable (than the planar conformation by 56.2 $\mathrm{kcal} / \mathrm{mol}$ ) in agreement with Böyükata and Güvenç. ${ }^{47}$ The trigonal bipyramidal structure is so stable such that even at $2000 \mathrm{~K}$ it is not distorted. It should be noted that from DFT computation the planar rhombus structure is $16.8 \mathrm{kcal} / \mathrm{mol}$
TABLE IV. Average interatomic distance $d_{\langle n n\rangle}$ (in angstrom) of small $\mathrm{Al}_{x}$ clusters used in the training set. $c-s$ means the average distance from the atom in the center of the icosahedral to that on the surface.

\begin{tabular}{lccl}
\hline \hline Cluster & DFT & ReaxFF & \multicolumn{1}{c}{ Others } \\
\hline $\mathrm{Al}_{2}$ & 2.636 & 2.585 & $2.51^{\mathrm{a}}$ \\
$\mathrm{Al}_{3}$ & 2.524 & 2.583 & $2.51,2.62^{\mathrm{b}}$ \\
$\mathrm{Al}_{6}$ & 2.724 & 2.525 & $2.6975^{\mathrm{a}}$ \\
$\mathrm{Al}_{13}$ & 2.814 & 2.842 & \\
$\mathrm{Al}_{13}(c-s)$ & 2.672 & 2.700 & \\
\hline
\end{tabular}

${ }^{\mathrm{a}}$ Reference 9

${ }^{\mathrm{b}}$ Reference 2

more stable than the trigonal bipyramidal structure. In DFT the trigonal bipyramidal isomer is found to optimize in the hextet state. In the doublet configuration it transforms into a rhombus structure, whereas in the quartet state it transforms into a square pyramidal structure [Fig. 3(e)]. Another interesting finding is in the isomers of $\mathrm{Al}_{7}$. DFT predicts that the capped trigonal antiprism $\left(D_{3 d}\right)$ [Fig. 3(f)] is the most stable isomer, in agreement with the works of Jones ${ }^{49}$ and Jug et $a l .{ }^{49}$ However, Böyükata and Güvenç ${ }^{47}$ predicted that a pentagonal bipyramidal structure [Fig. 3(g)] was the most stable. In agreement with the latter, we found that the pentagonal bipyramidal structure was more stable than the capped trigonal antiprism by $2.3 \mathrm{kcal} / \mathrm{mol}$. However, this energy difference is quite small. Interestingly, the pentagonal bipyramidal structure retained its shape when optimized in VASP in the hextet but transformed to capped trigonal antiprism in the doublet and quartet states. This suggests that it is a higher excited isomer of $\mathrm{Al}_{7}$. DFT predicts that the capped trigonal antiprism is more stable than the pentagonal bipyramidal structure by $44.9 \mathrm{kcal} / \mathrm{mol}$.

The stability of the planar isomers of $\mathrm{Al}_{4}$ and $\mathrm{Al}_{5}$ over their 3D counterparts and of capped trigonal antiprism in the case of $\mathrm{Al}_{7}$ is a quantum mechanical effect inherent in DFT due to exchange correlation. If the overlap of the orbitals is not considered in DFT, then the 3D isomers should be preferred. This explains the discrepancy between ReaxFF and DFT in the case of these clusters. From a force field point of view the stability of the pentagonal bipyramidal isomer of $\mathrm{Al}_{7}$ over the capped trigonal antiprism is because the former is more symmetric than the latter. In the force field approach the higher the symmetry, the more stable the structure. In the case of DFT/ab initio, on the other hand, orbital overlaps and exchange-correlation effects play a crucial role in determining the stability of structures, which leads to isomers that are not necessarily highly symmetric being more stable.

In agreement with Ref. 47, both ReaxFF and DFT show that a capped pentagonal bipyramidal structure is the most stable isomer of $\mathrm{Al}_{8}$. For $8 \leq n<13$ clusters the pentagonal ring forms the backbone. Starting from $\mathrm{Al}_{11}$ onward there is at least one internal atom coupled with bulklike coordination. $\mathrm{Al}_{12}$ has two pentagonal rings. The lowest energy structure can be thought of as being made from $\mathrm{Al}_{13}$ by removing an atom without changing the icosahedral symmetry. The most stable isomer of $\mathrm{Al}_{14}$ is formed by capping of one face of the 


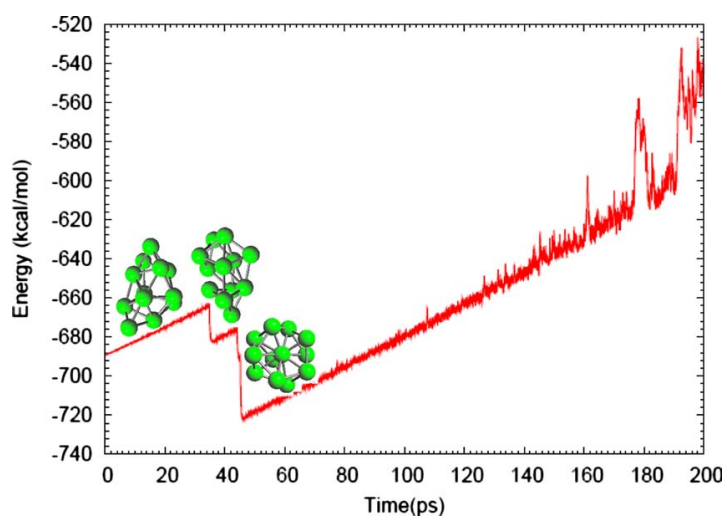

FIG. 4. (Color online) Heating up of $\mathrm{Al}_{12}$ at a rate of $2.5 \times 10^{9} \mathrm{~K} / \mathrm{s}$.

icosahedral structure of $\mathrm{Al}_{13}$. $\mathrm{Al}_{19}$ is made of two icosahedral structures fused together. Even at $\mathrm{Al}_{55}$ the icosahedral coordination is retained.

From the foregoing analysis one might wonder that if in some cases ReaxFF gives results for small clusters that differ significantly from those of DFT, why then do the two agree for larger clusters? What is it about the size that gives rise to this? Can we be so confident about the force field in spite of the significant difference in prediction between ReaxFF and DFT for small clusters? We reiterate that ReaxFF results are in line with predictions of other potentials. ${ }^{47,50,51}$ In the atomistic based-potential approach stability of structures are dictated by the number of bonds and symmetry. In DFT other effects such as Jahn-Teller distortion and spin polarization play a role in determining the most stable configuration. ${ }^{9,52}$ For instance, in DFT and other experiments $\mathrm{Al}_{2}$ has two isomers, but from a force field perspective $\mathrm{Al}_{2}$ has only one conformation. An extensive investigation of the isomers of $\mathrm{Al}_{n}$ up to $n=10$ has been given by Jones. ${ }^{46}$ Table IV shows the average interatomic distances for selected aluminum clusters as computed by DFT and ReaxFF in comparison to previous studies.

Now, as mentioned earlier, determining the global minima for clusters using simulated annealing is a delicate task since there are bound to be several minima in the potential energy hypersurfaces. Therefore, it should be noted that using a slow heating rate is important since this enables the system to have enough time to sample the various possible conformations in phase space. Careful analysis and tests showed that a heating rate of $2.5 \times 10^{9} \mathrm{~K} / \mathrm{s}$ was capable of predicting the most stable isomers of the well known isomers of small aluminum clusters, while if a heating rate of $2.5 \times 10^{10} \mathrm{~K} / \mathrm{s}$ was used some of these isomers were missed during the simulated annealing process. For instance, at a heating rate of $2.5 \times 10^{10} \mathrm{~K} / \mathrm{s}$ we could not capture the stable isomer of $\mathrm{Al}_{12}$. When the heating rate was reduced to $2.5 \times 10^{9} \mathrm{~K} / \mathrm{s}$ it was located by the force field. This is illustrated in Fig. 4.

This shows that it is important to use a well optimized heating rate in order to avoid being trapped in an energetically unfavorable local minimum. This point is also noted by Zhang et al. ${ }^{53}$ who concluded in their work on aluminum clusters with around 55 atoms that in the limit of long equilibration time the system will anneal into the lowest energy structure prior to melting.

Another important point is that the temperature at which the structure is equilibrated determines how soon the system locates its global minimum. As an example, the most stable form of $\mathrm{Al}_{13}$ cluster is an icosahedral $\left(I_{h}\right)$ structure. To determine whether ReaxFF can reproduce this we took a distorted $\mathrm{Al}_{13}$ cluster and heated it up at various temperatures, viz., 500, 1000, and $1500 \mathrm{~K}$. Figure 5 shows the results of the simulation. At $500 \mathrm{~K}$ the system is trapped in a local minimum and the resulting structural modification is not the most stable.

However, by doing simulated tempering at an elevated temperature of $1000 \mathrm{~K}$ more phase space becomes accessible to the system and the icosahedral configuration is captured by the system. The system finds a global minimum at a much earlier time at a temperature of $1500 \mathrm{~K}$ as compared to 1000 $\mathrm{K}$, since more phase space is accessible to the system at earlier time at this temperature. Thus an increase in temperature makes more phase space to be available to the system. At $500 \mathrm{~K}$ the icosahedral configuration is not accessible to the system within the time scale of the simulation.

To investigate the relationship between clusters and their relative stability several aluminum clusters $N \leq 108$ were cut from a periodic crystal. The clusters were then optimized using the force field optimization engine. To further reduce the bad contact angles the clusters were minimized using low temperature $(1 \mathrm{~K}) \mathrm{MD}$ simulation for 20000 steps (where one time step is $1 \mathrm{fs}$ ). This was followed by an equilibration run at $300 \mathrm{~K}$ for 100000 time steps. After this, each of these clusters was heated up to $2000 \mathrm{~K}$ at a rate of 2.5 $\times 10^{9} \mathrm{~K} / \mathrm{s}$ for 800000 steps in a NVT simulation using Berendsen thermostat. ${ }^{54}$ This was followed up by equilibration at this temperature for further 200000 steps. The equilibrated structures were then slowly annealed to $0 \mathrm{~K}$ at a rate of $2.5 \times 10^{9} \mathrm{~K} / \mathrm{s}$. This process was repeated four times but each time at a different temperature, viz., 300, 500, 600, and $800 \mathrm{~K}$. The internal energy values from the annealed geometries were then averaged. Figure 6 shows the second finite difference of the total energy (stability function) with respect to the cluster size $N$, which is defined as

$$
S(N)=E_{\mathrm{tot}}(N+1)+E_{\mathrm{tot}}(N-1)-2 E_{\mathrm{tot}}(N) .
$$

The peaks in the figure indicate that the clusters are quite stable (magic clusters), while the minima correspond to the most unstable structures. We can see in Fig. 6 that $\mathrm{Al}_{13}, \mathrm{Al}_{19}$, $\mathrm{Al}_{23}$, and $\mathrm{Al}_{55}$ are magic clusters. The fact that the force field is able to capture these very stable clusters gives further confidence in the suitability of $\operatorname{ReaxFF}_{\mathrm{Al}}$ in modeling aluminum metal. Our predictions of magic clusters of aluminum is consistent with the work of Doye. ${ }^{14}$ Doye predicted that $\mathrm{Al}_{44}$, $\mathrm{Al}_{61}, \mathrm{Al}_{67}, \mathrm{Al}_{69}$, and $\mathrm{Al}_{72}$ are magic clusters. This is consistent with $\mathrm{ReaxFF}_{\mathrm{Al}}$ 's prediction. One major area of disagreement with Doye is that he predicted $\mathrm{Al}_{48}$ to be a stable cluster, whereas $\mathrm{ReaxFF}_{\mathrm{Al}}$ predicts $\mathrm{Al}_{49}$ to be a stable structure. $\mathrm{ReaxFF}_{\mathrm{Al}}$ prediction is consistent with the well known magic clusters. ${ }^{55}$ The stability of these clusters from a geometrical approach has to do with their high symmetry in comparison 

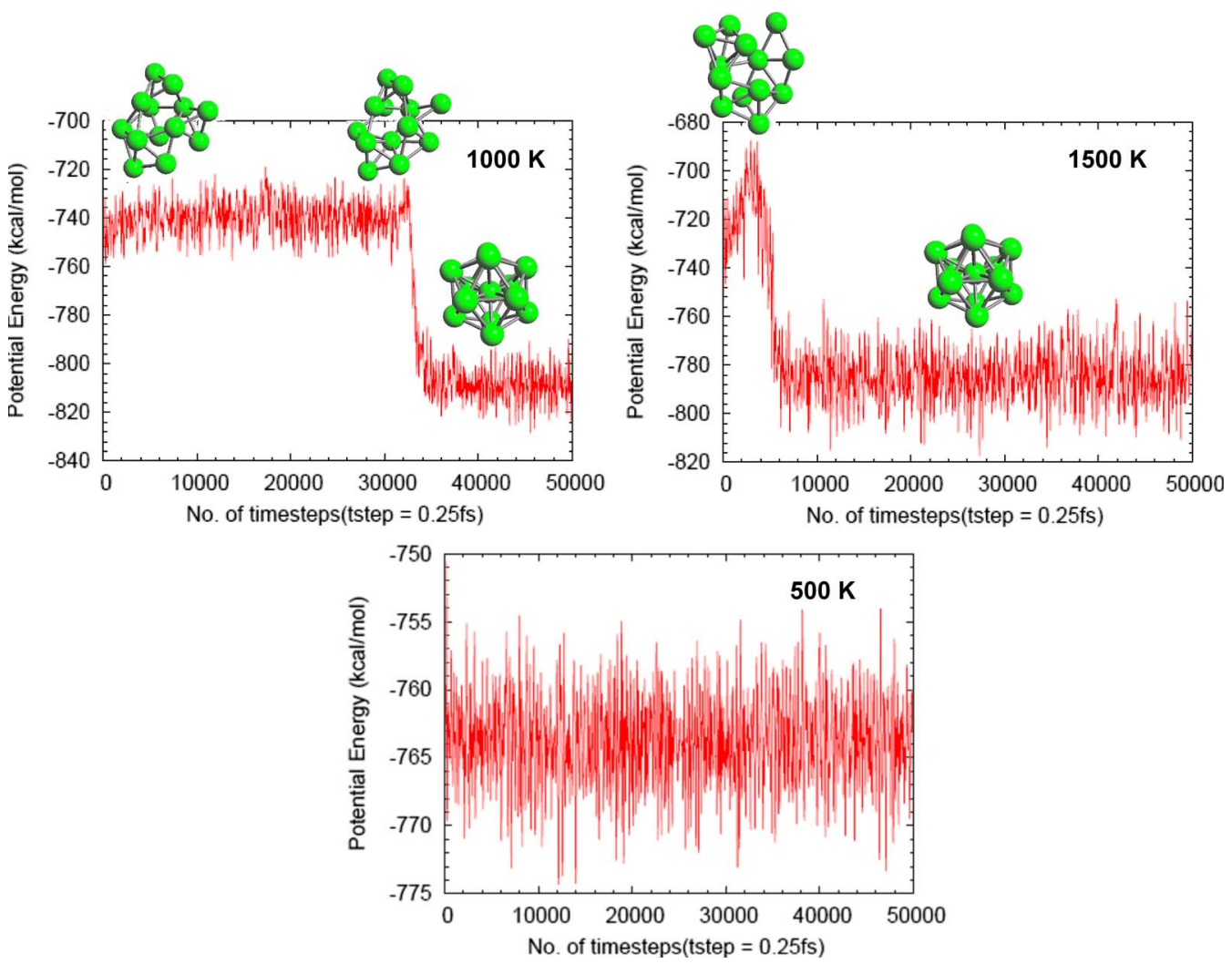

FIG. 5. (Color online) Simulated tempering of $\mathrm{Al}_{13}$ cluster.

to neighboring clusters along the stability energy surface. Joswig and Springborg, ${ }^{50}$ using Sutton-Chen ${ }^{56}$ potential, found the stable clusters to be those with $n=4,6,12,14,17$, $21,23,30,39,42,45,49$, and 56 atoms, whereas those with $n=5,11,13,15,22,28,33,36,41,44,46,51,53$, and 55 atoms were found to be particularly unstable. The authors, however, had some reservations on their results especially in view of the fact that $\mathrm{Al}_{13}$, from $a b$ initio computations, is a well known magic cluster. Figure 7 shows the annealed structures of $\mathrm{Al}_{13}, \mathrm{Al}_{19}, \mathrm{Al}_{23}, \mathrm{Al}_{55}, \mathrm{Al}_{67}$, and $\mathrm{Al}_{72} . \mathrm{Al}_{13}$ has one atom in the middle, whereas $\mathrm{Al}_{19}$ can be thought of as being made up of two $\mathrm{Al}_{13}$ clusters that have been fused together in such a way that there is an atom at the center of each half of the fused cluster.

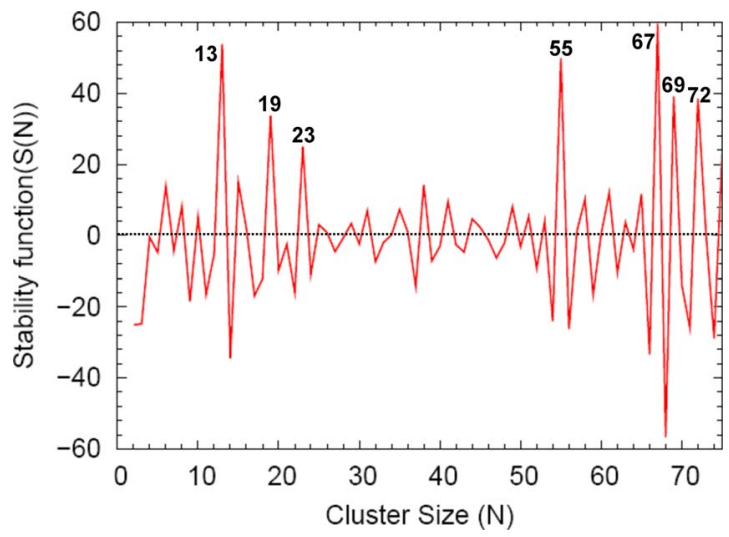

FIG. 6. (Color online) Stability function as a function of cluster size. The peaks in the figure show the most stable clusters based on geometric considerations. Positive values of stability function indicate that the cluster is stable.
The overall binding energy for a cluster can be partitioned into bulk, surface, edge, and corner contributions as follows:

$$
E_{\text {coh }}=E_{\text {bulk }}+a_{\text {surf }} N^{-1 / 3}+a_{\text {edge }} N^{-2 / 3}+a_{\text {corner }} N^{-1} .
$$

This approximation is valid provided that geometrical effects dominate and the electronic shell structure contributions are negligible. This is true especially for larger clusters whose stabilities are purely a function of structural configurations. Figure 8 shows a graph of binding energy as a function of $N^{-1 / 3}$. In general the edge and corner contributions are negligible especially in the case of aluminum where the clusters tend to have a spherical shape. These two contributions might become important in the case of very small clusters.
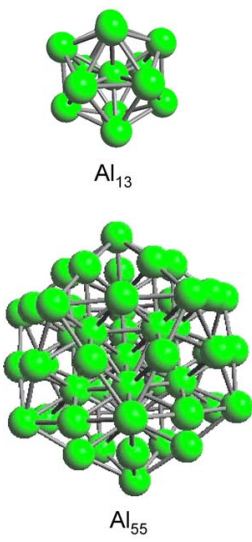
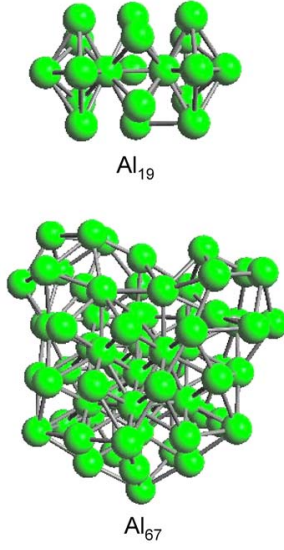
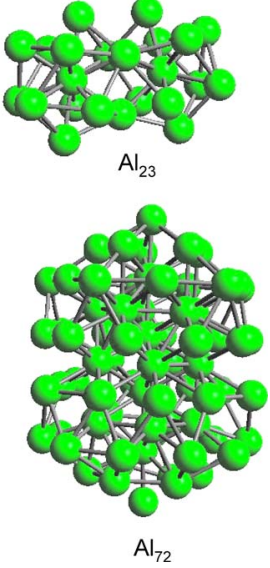

FIG. 7. (Color online) Some of the magic clusters of aluminum, $\mathrm{Al}_{13}, \mathrm{Al}_{19}$, $\mathrm{Al}_{23}, \mathrm{Al}_{55}, \mathrm{Al}_{67}$, and $\mathrm{Al}_{72}$, predicted by ReaxFF. 


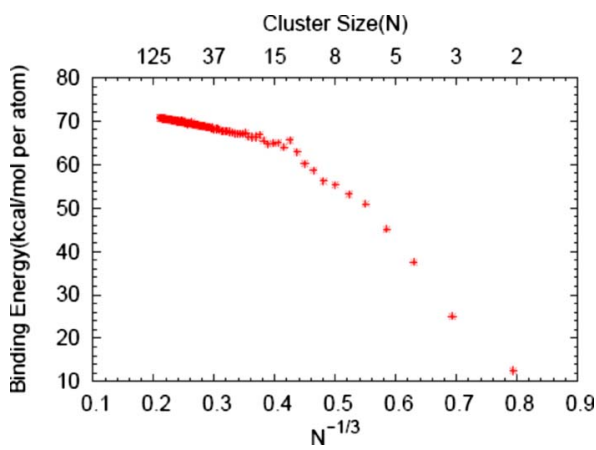

FIG. 8. (Color online) Binding energy per atom for aluminum clusters with $N=2,3,4,5, \ldots, 108$ as a function of cluster size $(n)$.

By a linear regression analysis (in which we considered only clusters with $N \geq 20$ ), the bulk binding energy is estimated to be

$$
E_{\text {bulk }}=76.8 \mathrm{kcal} / \mathrm{mol} \text {. }
$$

This is consistent with the experimental value of $77.48 \mathrm{kcal} /$ mol (Ref. 57) and that of Ahlrichs and Eliiot ${ }^{52}$ who found a value of $77.25 \mathrm{kcal} / \mathrm{mol}$. One important thing is that the relationship in Eq. (5) should only be applied to clusters of comparable structure. If we only consider clusters for $N$ $\geq 80$, then $E_{\text {bulk }}=76.85 \mathrm{kcal} / \mathrm{mol}$, which is still close to that in Eq. (6). The domination of surface energy contribution at low values of $N$ can be seen from the fact that for $N \leq 13$ the value of $a_{\text {surf }}$ term is $-141.33 \mathrm{kcal} / \mathrm{mol} N^{-1 / 3}$. This term drops significantly to a value of $-28.426 \mathrm{kcal} / \mathrm{mol} N^{-1 / 3}$ for $N \geq 20$. Thus surface energy contribution plays a dominant role for very small clusters.

\section{B. Melting and icosahedra to fcc transition}

Dynamic coexistence of solid and liquid phases for small clusters $^{58}$ makes it difficult to pinpoint with exactitude the melting point. In particular, for clusters there are fluctuations in potential energy with respect to temperature at the solid/ liquid interface. This is due to premelting, which arises from surface effects. In the bulk scenario melting is accompanied by an abrupt change in potential energy so that there is a clearly defined solid to liquid transition. In ideal experimental situation there is always some surface. Therefore to determine the bulk melting point we used an aluminum slab with five layers. The dimensions of the Al (111) slab were $(28.6 \times 24.75)$, giving a total of 500 atoms. A vacuum equivalent to five layers was used in the $z$ direction. This suitably separated the slab from its periodic images. The system was first equilibrated at $300 \mathrm{~K}$ and then heated up at a rate of $2.5 \times 10^{9} \mathrm{~K} / \mathrm{s}$. The results of the computation are shown in Fig. 9.

As can be seen in Figs. 9(a) and 9(b), the melting transition is accompanied by a rapid increase in energy. If we take the melting temperature to be the center on the peak in the heat capacity, then we get a melting temperature of 1240
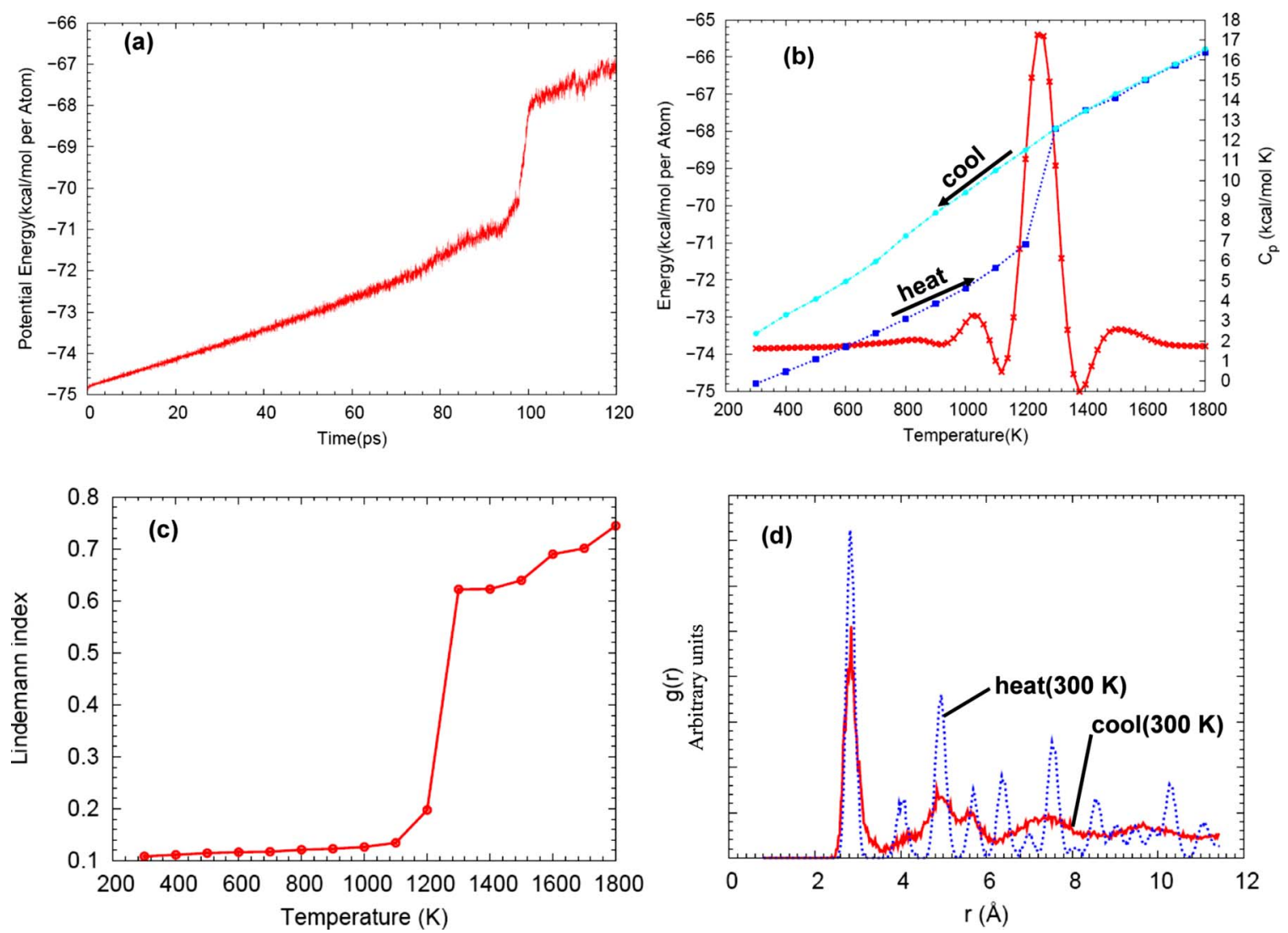

FIG. 9. (Color online) (a) Variation in potential energy with time during the heating process of aluminum slab with 500 atoms. (b) Potential energy and heat capacity $C_{p}(T)$ for heating and cooling cycle of aluminum slab with 500 atoms. (c) The Lindemann index for heating aluminum slab with 500 atoms. (d) The RDFs of the initial starting structure at $300 \mathrm{~K}$ and the cooled structure at $300 \mathrm{~K}$. From the RDFs, the starting structure is crystalline but the cooled structure is in an amorphous state (indicated by a split in the second peak). 

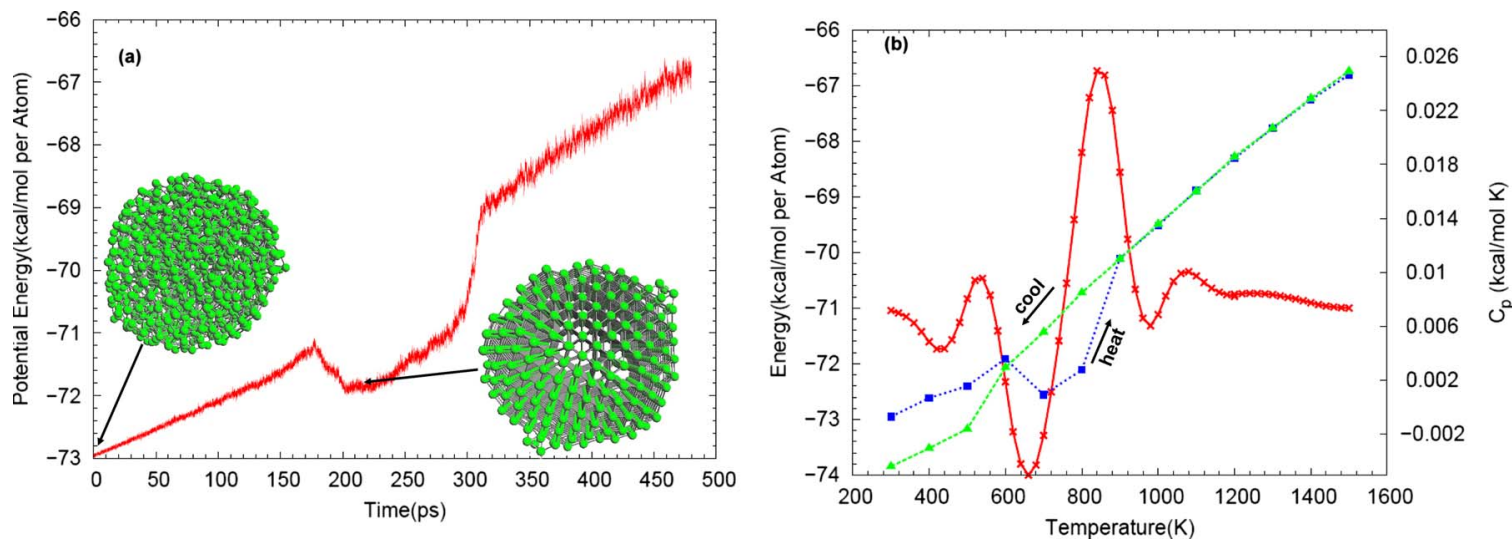

FIG. 10. (Color online) (a) Variation in potential energy with time during the heating process of aluminum cluster with 1024 atoms. The starting structure is amorphous. At about $170 \mathrm{ps}(700 \mathrm{~K})$ the system finds a more stable configuration, which is crystalline. (b) Potential energy and heat capacity $C_{p}(T)$ for heating-cooling cycle of aluminum cluster with 1024 atoms. When cooled, the system goes to a crystalline state.

$\mathrm{K}$. This is consistent with the Lindemann index criterion [Fig. 9(c)]. In Fig. 9(b), the cooled structure is less stable than the starting structure. This means that the system undergoes a phase transformation into a less stable (amorphous) structure. The radial distribution functions (RDFs) of the starting and the end (cooled) structures are shown in Fig. 9(d). Amorphization occurs because the cooling rate is fast and as such the system does not have enough time for atomic rearrangement into a crystalline structure. This is why the energy of the cooled structure is higher than that of the starting structure. An interesting observation on bulk melting is given by Lutsko et al. ${ }^{59}$ They suggested the introduction of a factor between the simulated and the experimental melting points when periodic boundary conditions are imposed for bulk systems. The experimental melting point is taken to be 0.75-0.85 times its simulated counterpart. Strictly speaking, the criteria of Lutsko et al. ${ }^{59}$ applies to a superheating required to melt a perfect crystal in the 3D periodic simulations. Superheating in ideal periodic simulations is related to the fact that only the homogeneous nucleation mechanism is available in the simulations. In a surface slab simulation, the surface induced melting mechanism is available, and for a properly equilibrated simulation superheating should not be observed. Since we used a slab approach, the criteria of Lutsko et al. ${ }^{59}$ does not hold.

Unlike bulk situation, in clusters, due to surface effects, there are no abrupt changes in the calorific curve, which normally is an indication of a phase transition from solid to liquid state. This presents a challenge in locating the exact melting point of clusters. Moreover, the phenomenon of dynamic coexistence melting implies that melting occurs over a range of temperature. A popular way to circumnavigate this problem is by equilibrating the clusters at various specific temperatures and then computing the thermodynamical and structural properties at each respective state. Figure 10 shows the potential energy scans for aluminum cluster with 1024 atoms. It can be seen in the figure that there is hysteresis between the heating and cooling graphs due to entropyinduced supercooling.

What is interesting in Fig. 10(a) is the fall in potential energy at about $170 \mathrm{ps}$, which is due to the system finding a more stable configuration whose annealed crystalline geom- etry is shown in the figure. We shall discuss the local ordering in this stable structure later. Looking at Fig. 10(b), we see that the heat capacity has negative values. Since this was not a microcanonical simulation, the negative heat capacity implies that the system was initially in a metastable state but found a more stable configuration prior to melting. This led to a fall in potential energy and concomitantly gave rise to a negative heat capacity. At around $310 \mathrm{ps}$ the potential energy of the system rises up rapidly due to melting. The calculated melting point for this representative configuration is $840 \mathrm{~K}$. In the cooling stage the cluster finds a lower minimum than the starting structure. The geometry of this minimum is crystalline. This implies that the cooling rate was slow enough so that the atoms had enough time to diffuse into their equilibrated positions and crystallize. The heating and cooling graphs intersect at 620 and $900 \mathrm{~K} .620 \mathrm{~K}$ is the threshold temperature for the crystallization of the supercooled structure, while $900 \mathrm{~K}$ is very close to the melting point of the cluster. Figure 11 shows the RDFs of the $\mathrm{Al}_{1024}$ clusters at 1500 and $300 \mathrm{~K}$ (upon cooling).

There is a very strong dependence of the melting point on the starting configuration. This is illustrated in Fig. 12, which shows the caloric and heat capacity curves for the case, whereby the annealed stable configuration in Fig. 12(a) is used as the starting structure in Fig. 12(b).

What is evident is that in this instance there is no nega-

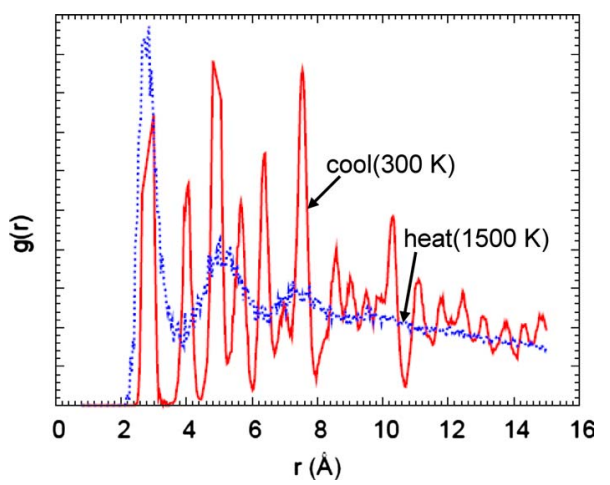

FIG. 11. (Color online) The radial distribution functions of the heated and cooled conformations of aluminum cluster with 1024 atoms, as shown in Fig. 10. 

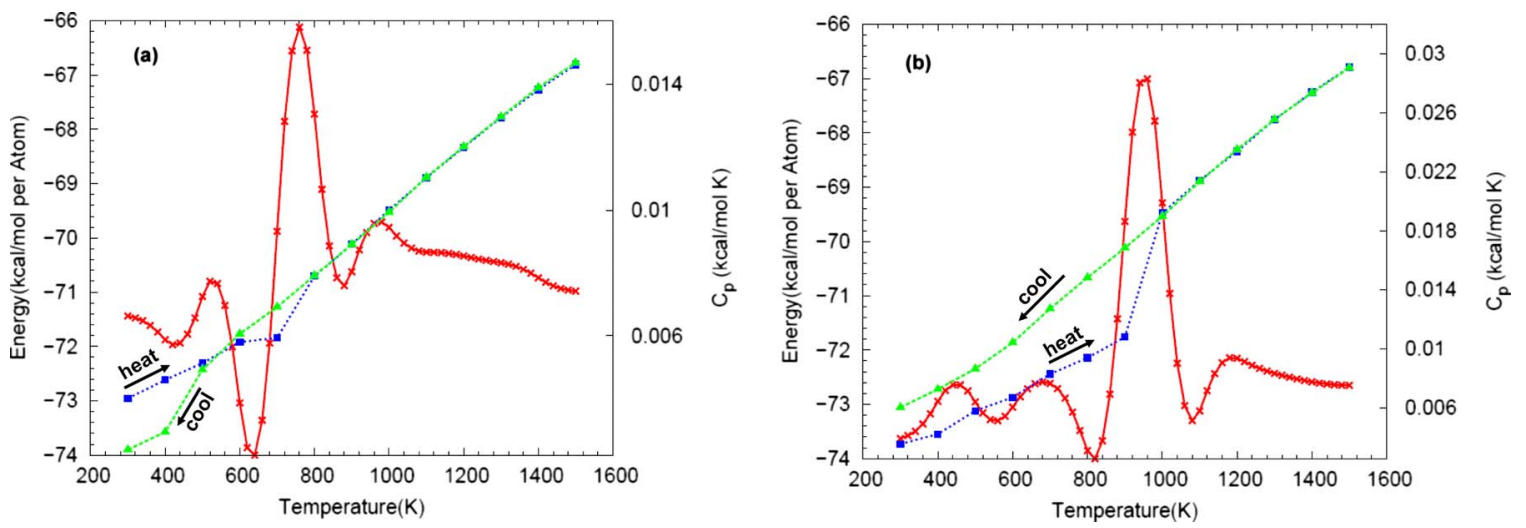

FIG. 12. (Color online) The potential energy and heat capacity $C_{p}(T)$ for heating-cooling cycle of aluminum cluster with 1024 atoms starting with different configurations.

tive heat capacity although the starting configuration in Fig. 12(a) is amorphous. The annealed configuration in Fig. 12(a) was used as the starting configuration in Fig. 12(b). What is interesting is that although the cooling rate in Fig. 12(b) is the same as that in Fig. 12(a), the structure, in the former figure, goes to an amorphous configuration. Actually it is as if it goes back to the starting configuration in Fig. 12(a). The calculated melting points in Figs. 12(a) and 12(b) are 760 and $960 \mathrm{~K}$, respectively. Note that the starting structure in Figs. 10 and 12(a) goes to the same crystalline state when annealed, which is the starting structure in Fig. 12(b). The high melting point of Fig. 12(b) shows that this configuration is the most stable.

It should be reiterated that one must be very careful when talking about the melting point of clusters. Clusters can have many isomers and the system can be trapped in any one of these accessible metastable configurations. Therefore the ideal melting point of a cluster should be an average of all the accessible states. However, the theoretical melting points of clusters in the literature are those for the most stable configurations. Table $\mathrm{V}$ shows the melting point of $\mathrm{Al}_{256}$ and that of the bulk as computed by various potentials in Ref. 60 and ReaxFF. ReaxFF values were computed using a heating rate of $2.5 \times 10^{8} \mathrm{~K} / \mathrm{s}$. The bulk experimental value $(933 \mathrm{~K})$ is also given.

It can be seen that ReaxFF gives a bulk melting point $(1240 \mathrm{~K})$ that is in excellent agreement with the glue potential, ${ }^{61}$ but higher than that in EAM (Ref. 48) and Streitz-Mintmire ${ }^{62}$ potentials by about $100 \mathrm{~K}$. Using the glue, EAM, Streitz-Mintmire, and Sutton-Chen ${ }^{56}$ potentials

TABLE V. Melting point of $\mathrm{Al}_{256}$ and bulk aluminum as computed by various potentials (Ref. 60) and ReaxFF. The given values for ReaxFF were those determined from a heating rate of $2.5 \times 10^{8} \mathrm{~K} / \mathrm{s}$.

\begin{tabular}{lcc}
\hline \hline Method & $\mathrm{Al}_{256}$ & Bulk \\
\hline Glue $^{\mathrm{a}}$ & 473 & 1244 \\
EAM & 448 & 1146 \\
Streitz-Mintmire & 448 & 1146 \\
Sutton-Chen & 400 & 529 \\
Expt. & & 933 \\
ReaxFF & 800 & 1240 \\
\hline
\end{tabular}

${ }^{\mathrm{a}}$ Reference 61 . the melting points of $\mathrm{Al}_{256}$ were calculated to be 473,448 , 448 , and $400 \mathrm{~K}$, respectively. ReaxFF, on the other hand, gives a melting point of $800 \mathrm{~K}$. However, for clusters of this size there is dynamical coexistence melting and as such it is quite challenging to pinpoint the exact melting point. There are no experimental works to validate our results. However, Breaux et $a l .{ }^{63}$ experimentally determined the melting points for $\mathrm{Al}_{49}^{+}-\mathrm{Al}_{63}^{+}$to be in the range of $450-650 \mathrm{~K}$. In the work of Neal et al. ${ }^{64}$ they computed the melting point of aluminum clusters with 25-83 atoms to be in the range of 600-700 K. These results are therefore consistent with the previous works. Figure 13 shows the heating curve and RDFs for $\mathrm{Al}_{256}$. The $\mathrm{Al}_{256}$ cluster was heated up from 0 to $1250 \mathrm{~K}$ at a rate of $2.5 \times 10^{8} \mathrm{~K} / \mathrm{s}$.

In the figure, at around 600 ps there is a fall in the energy [region (a)], at a temperature of $200 \mathrm{~K}$, which implies that the system has found an energetically lower conformation in comparison to the starting structure. Upon further heating, the system again finds another lower minimum at approximately 2800 ps [region (b)], at the temperature of 800 K. Shortly afterward, at 3800 ps, the energy of the system rises up rapidly indicating that there is a transition from solid to liquid state. The geometries of the annealed structures of (a) and (b) are shown in Fig. 13 (i). The two lower minimum structures $[(\mathrm{a})$ and (b)] were annealed to $0 \mathrm{~K}$ at a rate of $2.5 \times 10^{8} \mathrm{~K} / \mathrm{s}$. From the energies of the annealed configurations, the structure at point (b) was found to be more stable than that at point (a) by $0.455 \mathrm{kcal} / \mathrm{mol}$ atom. This is a huge energy difference. Figure 13 (ii) shows the radial distribution functions of structures at point (a), (b), and the starting structure (start). The presence of a double peak in the second shell of the structure (start) shows that it is amorphous. Structures (a) and (b) are clearly crystalline. In fact Fig. 13 (i) clearly illustrates that determining the global atomic arrangement in a cluster is a tricky business. As noted earlier, the rate at which the structure is heated up determines whether it adequately samples the whole of the phase space, and therefore finds global minimum or it is trapped in a local minimum. At a faster heating rate of $2.5 \times 10^{9}$ the two structures (a) and (b) in Fig. 13 (i) were not seen.

Structures (a) and (b) in Fig. 13 (i) were first annealed to $300 \mathrm{~K}$ and then heated up at a rate of $2.5 \times 10^{8} \mathrm{~K} / \mathrm{s}$. During the heating process it was seen, from the potential energy 

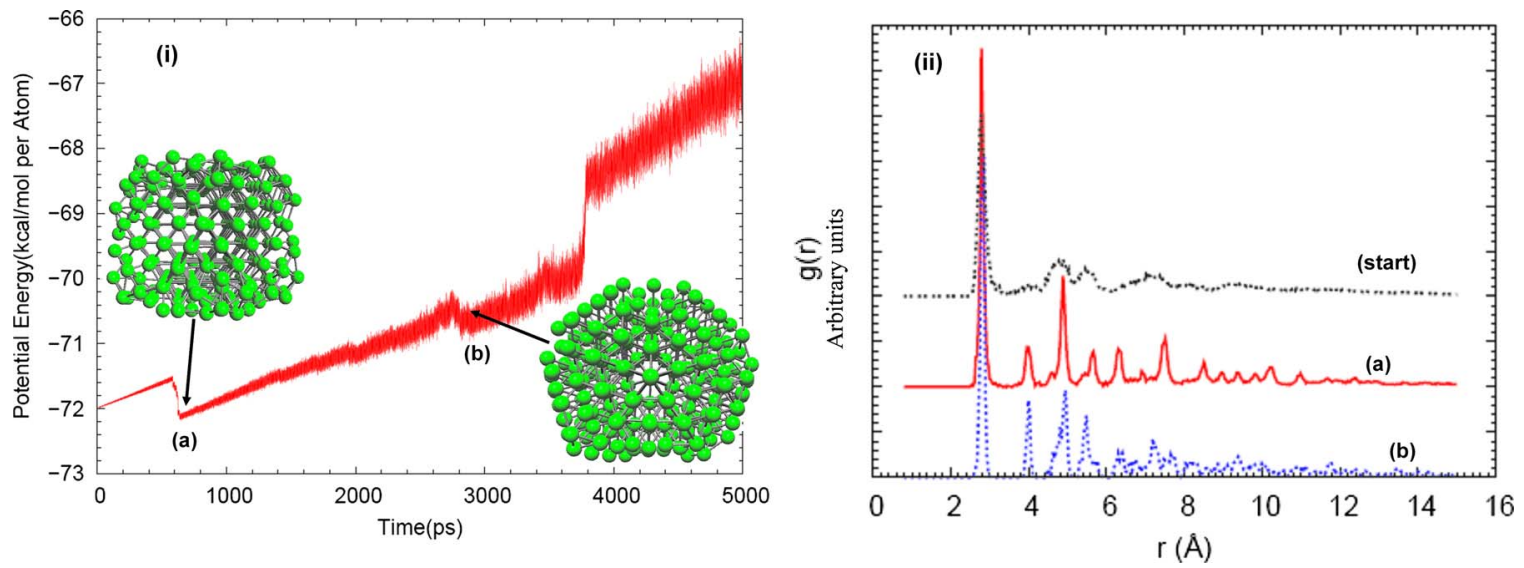

FIG. 13. (Color online) (i) Variation in energy with time during heating of $\mathrm{Al}_{256}$. The temperature was ramped up at a rate of $2.5 \times 10^{8} \mathrm{~K} / \mathrm{s}$. (ii) Radial distribution functions of structures (a), (b), and starting structure (start).

versus time curves, that structure (a) found other more stable conformations but structure (b) did not. Figure 14 shows the heating curve for structure (b).

It is clear in Fig. 14 (i) that there are no dips in potential energy arising as a consequence of the system finding other more stable conformations during the heating process. This indicates that indeed this structure might be the true minima. Figure 14 (ii) shows the RDFs of various points in Fig. 14 (i). While structure (b) shows some tendency toward amorphization, structures (c) and (d) exhibit liquidlike characteristics. Melting in small clusters of this size can be understood as follows. At the onset of melting the system spends most of its time in the solid phase but it intermittently swings to the liquid phase. Gradually as the temperature increases the system oscillates between the solid and liquid states. Further rise in temperature leads to the system spending most of its time in the liquid phase, although the solid phase is also present. What this tells us is that on melting a small system tries to avoid partially molten states by converting some of its kinetic energy into potential energy. As a result it keeps on oscillating between the solid and the liquid states as more and more energy is added to it. It is like the system is being driven toward liquid state by the addition of energy but it resists this force by converting some of its kinetic energy into potential energy thereby becoming colder and reverting back to the solid state. ${ }^{65}$ Care must be taken though since we are dealing with a canonical ensemble. However, for small clusters there exist wide fluctuations of temperature due to the finite cluster size. The overall temperature might remain constant but there can be local drops in temperature within certain regions of the cluster in tandem with the lowering of the potential energy. Eventually at a critical temperature $T^{\text {crit }}$ the system overcomes the transitional barrier to the pure liquid phase and spontaneously switches to the liquid state. This dynamic competition between the solid and liquid phases leads to a cluster size dependent semiliquid region, which is schematically shown in Fig. 15.

The smaller the size of the cluster, the larger is the width of the semiliquid region and vice-versa. A larger width implies that the system spends an appreciable amount of time in the liquid state before oscillating back to the solid state. In the bulk case the semiliquid phase does not exist.

One of the most fascinating issues when studying clusters is the threshold of bulk ordering as the size of the cluster increases. In order to understand the underlying nature of microscopic local atomic arrangement in structures (a) and (b) in Fig. 13 (i), we performed a HA pair analysis on the two structures during the cooling process. The results are shown in Fig. 16. In the following, in all HA pairs analysis,
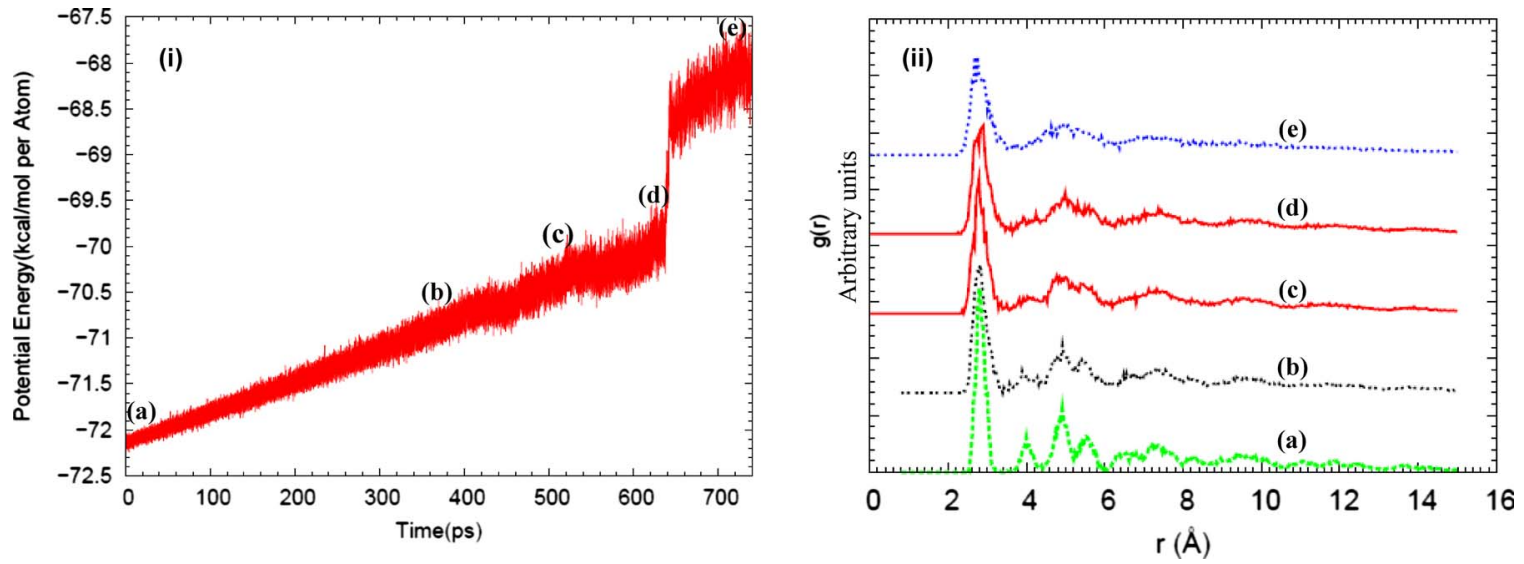

FIG. 14. (Color online) (i) The heating curve, at a heating rate of $2.5 \times 10^{8} \mathrm{~K} / \mathrm{s}$, for structure (b) in Fig. 13 (i). (ii) Radial distribution functions of structures (a), (b), (c), (d), and (e) formed during the heating process. 


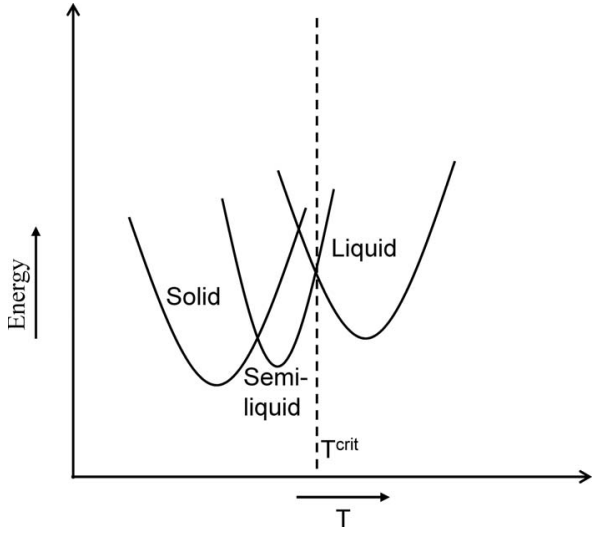

FIG. 15. Schematic representation of the structural evolution of a cluster with increase in temperature.

quantities have been normalized such that the total number of pairs considered $(1421,1422,1551$, and 1431) is unity.

Clearly, as can be seen in Fig. 16, in structure (a) the 1421 pairs, which are indicative of fcc ordering, dominate. Contributions from 1422, 1431, and 1551 pairs are negligible. The 1421 and 1422 pairs remain fairly constant during the cooling process. This implies that the fcc ordering is an inherent structure and cooling the system merely freezes the atoms at their equilibrium positions. Structure (b), on the other hand, has appreciable contributions from both 1421 and 1422 pairs, but the 1422 pairs are more dominant relative to the 1421 pairs. Therefore for a cluster of this size the most energetically favored geometry is the one with mixed hcp-fcc ordering. Figure 17 shows the HA pair analysis for the $\mathrm{Al}_{1024}$ cluster whose annealed crystalline geometry is shown in Fig. 10(a).

During the cooling process from 700 to $0 \mathrm{~K}$ the ratio of 1421 pairs to 1422 pairs remains quite high, $\approx 8: 1$. The 1551 and 1431 pairs are essentially 0 in this cluster throughout the cooling range. These high values of the 1421 pairs relative to the 1422 pairs throughout the whole cooling range show that for a cluster of this size the dominant domains are fcc.

Figure 18 shows the geometries of the $\mathrm{Al}_{3072}$ cluster. The amorphous configuration was the starting structure during the heating process. The crystalline configuration was a global minimum conformation located during the heating process. This configuration was then slowly cooled to $0 \mathrm{~K}$ at a rate of $2.5 \times 10^{9} \mathrm{~K} / \mathrm{s}$.

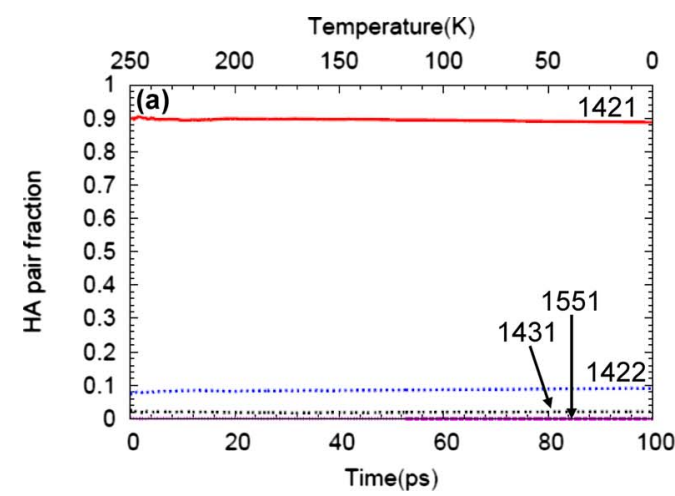

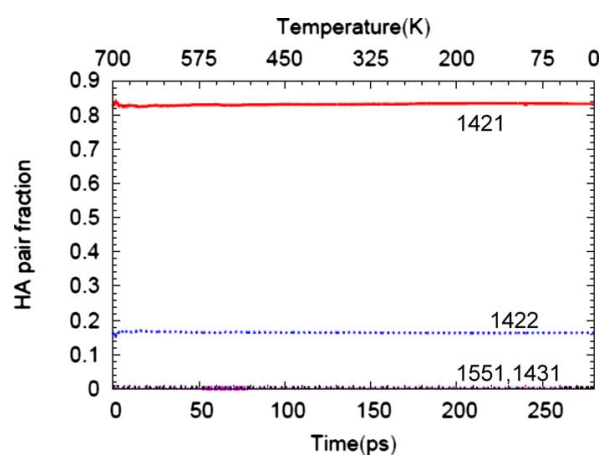

FIG. 17. (Color online) HA pairs for $\mathrm{Al}_{1024}$ during the cooling process from 700 to $0 \mathrm{~K}$.

The RDF of the two structures is shown in Fig. 19 in which a double peak on the second shell shows the amorphization of the starting configuration. Although the end configuration is crystalline the periodicity is irregular, which suggests that the ordering is not purely fcc but perchance an admixture of fcc and hep.

From the foregoing, a possible structural transformation scenario is icosahedral domains to hep domains then to mixed hcp/fcc ordering and lastly to fcc domains for the clusters nearing bulklike properties. There is an obvious interplay between favorable energy and the geometry of the cluster. For clusters with $N \leq 55$ icosahedral ordering is favored, for $256 \leq N<1024$ mixed hcp-fcc ordering is favored, while for $N \geq 1024 \mathrm{fcc}$ ordering is favored. From the respective potential energies at $0 \mathrm{~K}$ the cohesive energies per atom for $\mathrm{Al}_{55}, \mathrm{Al}_{108}, \mathrm{Al}_{256}, \mathrm{Al}_{512}, \mathrm{Al}_{1024}$, and $\mathrm{Al}_{3072}$ clusters are as shown in Table VI.

In Table VI the cohesive energy for $\mathrm{Al}_{3072}$ cluster is $-75.78 \mathrm{kcal} / \mathrm{mol}$ atom, which is quite close to the bulk cohesive energy $(76.8 \mathrm{kcal} / \mathrm{mol}$ atom $)$. This is why fcc ordering should dominate in this region.

\section{CONCLUSION}

A parametrized force field has been developed for aluminum systems. A detailed study of the energetics and optimized structures for aluminum clusters in the range of 2-108 atoms has been presented. In DFT computation spin polarization was taken into account, where it was seen that the lowest multiplicities were not necessarily the most stable.

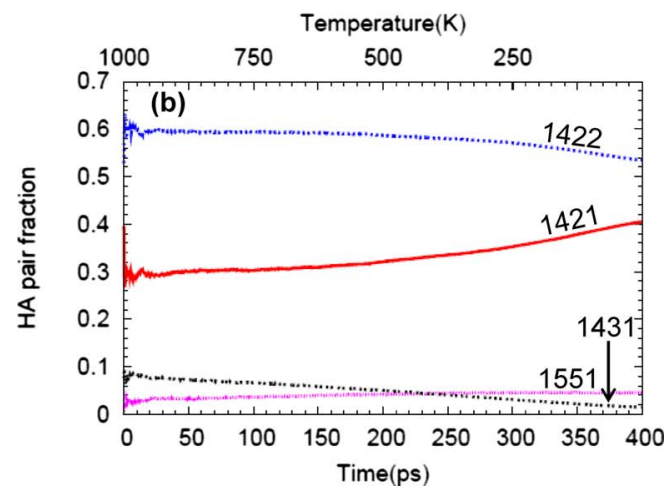

FIG. 16. (Color online) The relative number of bonded pairs, 1421, 1422, 1551, and 1431 for the two conformations of $\mathrm{Al}_{256}$ clusters (a) and (b), as shown in Fig. 13 (i). 

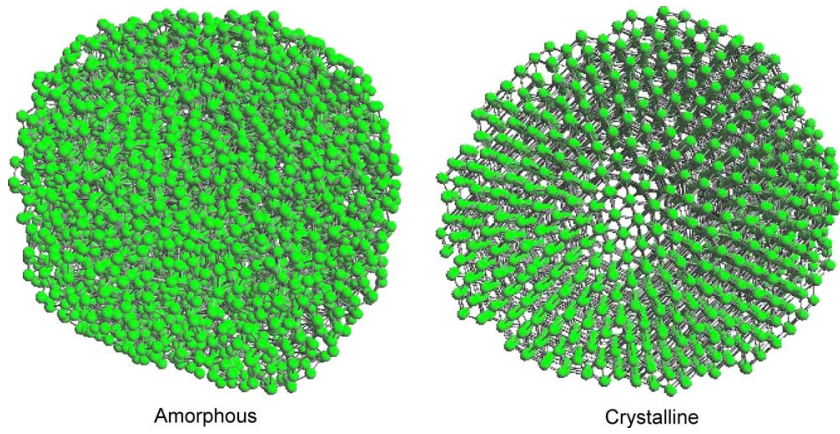

FIG. 18. (Color online) Geometries of $\mathrm{Al}_{3072}$.

Apart from $\mathrm{Al}_{4}$ and $\mathrm{Al}_{5}$ in which DFT prefers planar orientation but ReaxFF favors 3D structures, there is a general agreement between ReaxFF and DFT on the structures of aluminum clusters. The disparity between DFT and ReaxFF in these smaller clusters is due to quantum mechanical effect, whereby orbital overlap plays an important part in determining the most stable structure. As the size of the clusters increases the geometrical effects play a dominant role relative to electronic shell contributions. Therefore for larger clusters there is a good agreement between DFT and ReaxFF predictions. The results for the energetics, structural configurations, and magic numbers are in good agreement with both theoretical studies and experimental works. The bulk binding energy is computed to be $76.8 \mathrm{kcal} / \mathrm{mol}$, which is consistent with the experimental value of $77.48 \mathrm{kcal} / \mathrm{mol} .{ }^{57}$ This gives confidence in the suitability of ReaxFF for studying and understanding the underlying dynamics in aluminum clusters. In the quantification of the growth of cluster it is seen that as the size of the clusters increases there is preference for the coexistence of fcc/hcp orders at the expense of icosahedral ordering. It has been found that even for aluminum clusters with 512 atoms, surface effects are dominant and distorted icosahedral orders exist. Although it is well known that it is not easy to get to the global minima of a structure by doing simulated annealing, an important aspect of this research was the realization that it is possible to capture the global minimum of a structure by heating up the system very slowly. A possible structural transformation scenario is icosahedral domains to hcp domains then to mixed hcp/fcc ordering and lastly to fcc domains for the clusters nearing bulklike properties. There is an obvious interplay between favorable en-

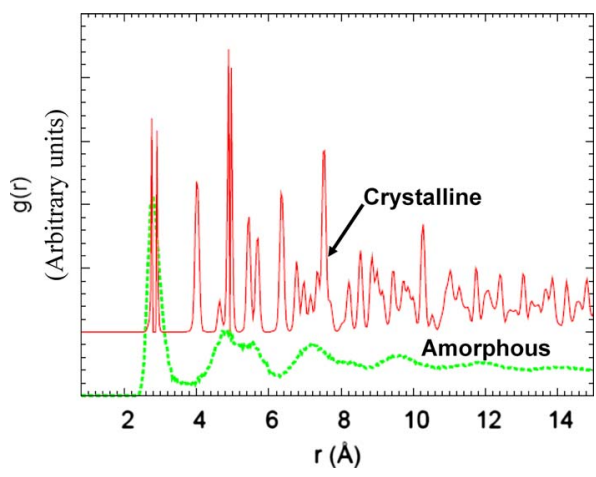

FIG. 19. (Color online) Radial distribution function of the amorphous and crystalline states of $\mathrm{Al}_{3072}$ cluster.
TABLE VI. Cohesive energies (in $\mathrm{kcal} / \mathrm{mol}$ per atom) for some aluminum clusters.

\begin{tabular}{ll}
\hline \hline Cluster & $E_{\text {coh }}$ \\
\hline $\mathrm{Al}_{55}$ & -69.62 \\
$\mathrm{Al}_{108}$ & -71.03 \\
$\mathrm{Al}_{256}$ & -72.33 \\
$\mathrm{Al}_{512}$ & -73.29 \\
$\mathrm{Al}_{1024}$ & -73.85 \\
$\mathrm{Al}_{3072}$ & -75.78 \\
\hline
\end{tabular}

ergy and the geometry of the cluster. For clusters with $N$ $\leq 55$ icosahedral ordering is favored, for $256 \leq N<1024$ mixed hcp-fcc ordering is favored, while for $N \geq 1024 \mathrm{fcc}$ ordering is favored.

\section{ACKNOWLEDGMENTS}

This work is part of the research programs of Advanced Chemical Technologies for Sustainability (ACTS), which is funded by Nederlandse Organisatie voor Wetenschappelijk Onderzoek (NWO). J.G.O.O. would like to thank Saman Alavi for help with the Lindemann index script.

${ }^{1}$ L. G. M. Pettersson, C. W. Bauschlicher, Jr., and T. Halicioglu, J. Chem. Phys. 87, 2205 (1987).

${ }^{2}$ T. H. Upton, J. Chem. Phys. 86, 7054 (1987).

${ }^{3}$ D. M. Cox, D. J. Trevor, R. L. Whetten, E. A. Rohlfing, and A. Kaldor, J. Chem. Phys. 84, 4651 (1986).

${ }^{4}$ W. A. de Heer, P. Milani, and A. Chtelain, Phys. Rev. Lett. 63, 2834 (1989).

${ }^{5}$ D. M. Cox, D. J. Trevor, R. L. Whetten, and A. Kaldor, J. Phys. Chem. 92, 421 (1988).

${ }^{6}$ J. Akola, M. Manninen, H. Häkkinen, U. Landman, X. Li, and L.-S. Wang, Phys. Rev. B 62, 13216 (2000).

${ }^{7}$ H.-P. Cheng, R. S. Berry, and R. L. Whetten, Phys. Rev. B 43, 10647 (1991).

${ }^{8}$ S. Debiaggi and A. Caro, Phys. Rev. B 46, 7322 (1992).

${ }^{9}$ S. H. Yang, D. A. Drabold, J. B. Adams, and A. Sachdev, Phys. Rev. B 47, 1567 (1993).

${ }^{10}$ J. Akola, H. Häkkinen, and M. Manninen, Phys. Rev. B 58, 3601 (1998).

${ }^{11}$ J.-Y. Yi, D. J. Oh, and J. Bernholc, Phys. Rev. Lett. 67, 1594 (1991).

${ }^{12}$ R. E. Leuchtner, A. C. Harms, and A. W. Castleman, Jr., J. Chem. Phys. 94, 1093 (1991).

${ }^{13}$ F. Ercolessi, M. Parrinello, and E, Tosatti, Philos. Mag. A 58, 213 (1988).

${ }^{14}$ J. P. K. Doye, J. Chem. Phys. 119, 1136 (2003).

${ }^{15}$ K. Joshi, D. G. Kanhere, and S. A. Blundell, Phys. Rev. B 67, 235413 (2003).

${ }^{16}$ Z.-Y. Lu, C.-Z. Wang, and K.-M. Ho, Phys. Rev. B 61, 2329 (2000).

${ }^{17}$ K. Joshi, D. G. Kanhere, and S. A. Blundell, Phys. Rev. B 66, 155329 (2002).

${ }^{18}$ S. Chacko, K. Joshi, D. G. Kanhere, and S. A. Blundell, Phys. Rev. Lett. 92, 135506 (2004).

${ }^{19}$ M. C. Payne, M. P. Teter, D. C. Allan, T. A. Arias, and J. D. Joannopoulos, Rev. Mod. Phys. 64, 1045 (1992).

${ }^{20}$ G. Kresse and J. Furthmüller, Phys. Rev. B 54, 11169 (1996).

${ }^{21}$ A. Strachan, A. C. van Duin, D. Chakraborty, S. Dasgupta, and W. A. Goddard, Phys. Rev. Lett. 91, 098301 (2003).

${ }^{22}$ A. C. T. van Duin, A. Strachan, S. Stewman, Q. Zhang, X. Xu, and W. Goddard III, J. Phys. Chem. A 107, 3803 (2003).

${ }^{23}$ Q. Zhang, T. Çağn, A. C. T. van Duin, W. A. Goddard, Y. Qi, and L. G. Hector, Phys. Rev. B 69, 045423 (2004).

${ }^{24}$ A. C. T. van Duin, S. Dasgupta, F. Lorant, and W. Goddard III, J. Phys. Chem. A 105, 9396 (2001)

${ }^{25}$ J. G. O. Ojwang, R. van Santen, G. Jan Kramer, A. C. T. van Duin, and W. A. Goddard III, J. Chem. Phys. 128, 164714 (2008).

${ }^{26}$ J. D. Honeycutt and H. C. Andersen, J. Phys. Chem. 91, 4950 (1987).

${ }^{27}$ S. Cheung, W.-Q. Deng, A. C. T. van Duin, and W. Goddard III, J. Phys. 
Chem. A 109, 851 (2005).

${ }^{28}$ J. Tersoff, Phys. Rev. Lett. 61, 2879 (1988).

${ }^{29}$ D. W. Brenner, Phys. Rev. B 42, 9458 (1990).

${ }^{30}$ C. R. Landis, T. Cleveland, and T. K. Firman, J. Am. Chem. Soc. 120, 2641 (1998)

${ }^{31}$ P. E. Blöchl, Phys. Rev. B 50, 17953 (1994).

${ }^{32}$ J. P. Perdew, J. A. Chevary, S. H. Vosko, K. A. Jackson, M. R. Pederson, D. J. Singh, and C. Fiolhais, Phys. Rev. B 46, 6671 (1992).

${ }^{33}$ J. P. Perdew, K. Burke, and Y. Wang, Phys. Rev. B 54, 16533 (1996).

${ }^{34}$ J. P. Perdew, K. Burke, and M. Ernzerhof, Phys. Rev. Lett. 77, 3865 (1996).

${ }^{35}$ H. J. Monkhorst and J. D. Pack, Phys. Rev. B 13, 5188 (1976).

${ }^{36}$ F. Birch, Phys. Rev. 71, 809 (1947).

${ }^{37}$ L. Verlet, Phys. Rev. 159, 98 (1967).

${ }^{38}$ F. A. Lindemann, Z. Phys. 11, 609 (1910).

${ }^{39}$ J. Jellinek and A. Goldberg, J. Chem. Phys. 113, 2570 (2000).

${ }^{40}$ W. K. Luo, H. W. Sheng, F. M. Alamgir, J. M. Bai, J. H. He, and E. Ma, Phys. Rev. Lett. 92, 145502 (2004).

${ }^{41}$ D. R. Nelson, Phys. Rev. Lett. 50, 982 (1983).

${ }^{42}$ Handbook of Chemistry and Physics, 82 nd ed., edited by D. R. Lide and E. Wiberg (CRC, New York, 2001).

${ }^{43}$ J. Koutecky, G. Pacchioni, G. H. Jeung, and E. C. Hass, Surf. Sci. 156, 650 (1985).

${ }^{44}$ K. Jug, H. Schluff, H. Kupka, and R. Iffert, J. Comput. Chem. 9, 803 (1988).

${ }^{45}$ C. W. Bauschlicher, Jr. and L. G. M. Pettersson, J. Chem. Phys. 84, 2226 (1986).

${ }^{46}$ R. O. Jones, J. Chem. Phys. 99, 1194 (1993).
${ }^{47}$ M. Böyükata and Z. B. Güvenç, Braz. J. Phys. 36, 720 (2006).

${ }^{48}$ M. S. Daw and M. I. Baskes, Phys. Rev. B 29, 6443 (1984).

${ }^{49}$ R. O. Jones, Phys. Rev. Lett. 67, 224 (1991).

${ }^{50}$ J.-O. Joswig and M. Springborg, Phys. Rev. B 68, 085408 (2003).

${ }^{51}$ L. Lloyd and R. Johnston, Chem. Phys. 236, 107 (1998).

${ }^{52}$ R. Ahlrichs and D. S. Eliiot, Phys. Chem. Chem. Phys. 1, 13 (1999).

${ }^{53}$ W. Zhang, F. Zhang, and Z. Zhu, Phys. Rev. B 74, 033412 (2006).

${ }^{54}$ H. J. C. Berendsen, J. P. M. Postma, W. F. van Gunsteren, A. Dinola, and J. R. Haak, J. Chem. Phys. 81, 3684 (1984).

${ }^{55}$ I. A. Harris, R. S. Kidwell, and J. A. Northby, Phys. Rev. Lett. 53, 2390 (1984).

${ }^{56}$ A. P. Sutton and J. Chen, Philos. Mag. Lett. 61, 139 (1990).

${ }^{57}$ M. F. Jarrod, in Clusters of Atoms and Molecules, edited by H. Haberland (Springer, Berlin, 1992), p. 293.

${ }^{58}$ S. Alavi and D. L. Thompson, J. Phys. Chem. A 110, 1518 (2006).

${ }^{59}$ J. F. Lutsko, D. Wolf, S. R. Phillpot, and S. Yip, Phys. Rev. B 40, 2841 (1989).

${ }^{60}$ P. Puri and V. Yang, J. Phys. Chem. C 111, 11776 (2007).

${ }^{61}$ F. Ercolessi and J. B. Adams, Europhys. Lett. 26, 583 (1994).

${ }^{62}$ F. H. Streitz and J. W. Mintmire, Phys. Rev. B 50, 11996 (1994).

${ }^{63}$ G. A. Breaux, C. M. Neal, B. Cao, and M. F. Jarrold, Phys. Rev. Lett. 94, 173401 (2005).

${ }^{64}$ C. M. Neal, A. K. Starace, and M. F. Jarrold, Phys. Rev. B 76, 054113 (2007).

${ }^{65}$ H. Haberland, in Atomic Clusters and Nanoparticles, edited by C. Guet, P. Hobza, F. Spiegelman, and F. David (Springer-Verlag, Berlin, 2001), pp. $32-55$. 